\title{
EFSUMB/WFUMB Webinar on Gastrointestinal Ultrasound
}

\section{September 201815.00 CEST}

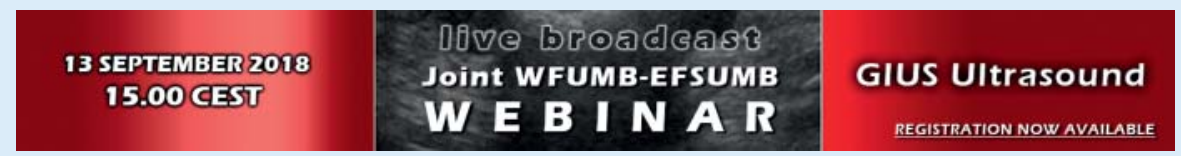

Odd Helge Gilja Chair

Kim Nylund, Norway: Basic methodology of GIUS and normal findings

Stephanie Wilson, Canada: Elastography of the bowels with particular reference to strictures Giovanni Maconi, Italy: Evaluation of deep remission in IBD using ultrasound

Carla Serra, Italy: CEUS of the bowels in IBD

\section{EFSUMB Webinar on Paediatric CEUS}

\section{October 201817.00 CET}

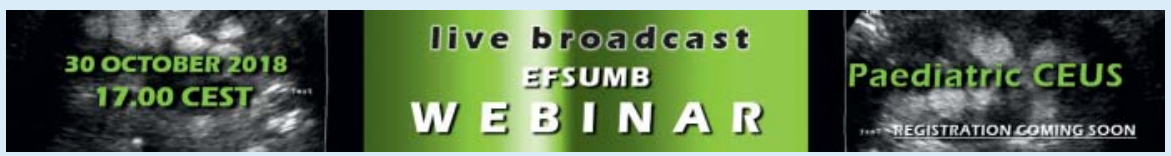

Paul Humphries Chair

Trauma: Liver VUR Oncology

Kasse Darge (USA), Annamaria Deganello (UK), Doris Franke (Germany) 